LAWRENCE LIVERMORE NAT IO N A L LABORATORY

\title{
Effective Operators in the NCSM Formalism
}

I. Stetcu, B. R. Barrett, P. Navratil, J. P. Vary

December 1, 2004

ENAM 04: The Fourth International Conference on Exotic Nuclei and Atomic Masses

Callaway Gardens, Pine Mountain, GA, United States September 12, 2004 through September 16, 2004 
This document was prepared as an account of work sponsored by an agency of the United States Government. Neither the United States Government nor the University of California nor any of their employees, makes any warranty, express or implied, or assumes any legal liability or responsibility for the accuracy, completeness, or usefulness of any information, apparatus, product, or process disclosed, or represents that its use would not infringe privately owned rights. Reference herein to any specific commercial product, process, or service by trade name, trademark, manufacturer, or otherwise, does not necessarily constitute or imply its endorsement, recommendation, or favoring by the United States Government or the University of California. The views and opinions of authors expressed herein do not necessarily state or reflect those of the United States Government or the University of California, and shall not be used for advertising or product endorsement purposes. 
EPJ manuscript No.

(will be inserted by the editor)

\title{
Effective Operators in the NCSM Formalism
}

\author{
Ionel Stetcu ${ }^{1}$, Bruce R. Barrett ${ }^{1}$, Petr Navrátil ${ }^{2}$, and James P. Vary ${ }^{3}$ \\ 1 Department of Physics, University of Arizona, P.O. Box 210081, Tucson, Arizona 85721, U.S.A \\ 2 Lawrence Livermore National Laboratory, Livermore, California 94551, U.S.A. \\ 3 Department of Physics and Astronomy, Iowa State University, Ames, Iowa 50011, U.S.A.
}

Received: date / Revised version: date

\begin{abstract}
No-core shell model (NCSM) calculations using ab initio effective interactions are very successful in reproducing the experimental nuclear spectra. While a great deal of work has been directed toward computing effective interactions from bare nucleon-nucleon (NN) and three-nucleon forces, less progress has been made in calculating the effective operators. Thus, except for the relative kinetic energy, the proton radius, and the NN pair density, all investigations have used bare operators. We apply the Lee-Suzuki procedure to general two-body operators, investigating the importance of the approximations involved. In particular we concentrate on the limitations of the two-body cluster approximation.
\end{abstract}

PACS. PACS-key 21.60.Cs - PACS-key 23.20.-g - PACS-key 23.20.Js

A long standing problem in the phenomenological shell model was the use of effective charges which arise, in principle, from the truncation of the space. Previous attempts to describe in perturbation theory phenomenological charges needed in the phenomenological shell model to obtain correct transition strengths have been unsuccessful [1], but more recent investigations within the framework of the no-core shell model (NCSM) have reported considerable success in predicting the large values of the effective charges [2].

In the NCSM, one starts from a nucleon-nucleon (NN) interaction which describes the NN scattering data, and one derives an effective interaction in a restricted model space. Three-body interactions have been shown to be important in the correct description of the energy spectra in light nuclei, but they are computationally very demanding so that in the current investigation we restricted ourselves to two-body interactions. As a result of the space truncation, one has also to compute effective operators.

The Lee-Suzuki transformation [3] has been used in order to accommodate the short range two-body correlations, with the condition that the model and excluded spaces are not coupled by the Hamiltonian. For details, we refer the reader to previous publications [4]. The same procedure can be applied to construct other effective operators [5].

In this paper, we obtain corrections to electromagnetic multipoles, and the results for a selected quadrupole transition strength are shown in Fig. 1. We point out that, because non-scalar operators can connect different channels with good angular momentum, the procedure to obtain effective operators is more involved than for the Hamiltonian. Therefore, for general one and two-body operators, one has to restrict the number of states from the $Q$-space included in the calculation [6], checking the convergence of the many-body matrix elements with the number of states included. In Fig. 1 we show how the renormalization of the $E 2$ operator varies with the number of states in the $Q$ space included in the calculation. The $B(E 2)$ remains almost constant, with negligible contributions from the states in the $Q$ space. This is somehow surprising, as the quadrupole operator connects the model space with the excluded space, and the renormalization was expected to improve the $B$ value obtained with the bare operator. The same result is obtained for the $M 1$ operator, but this result is easier to understand as this operator is defined completely in the model space. Calculation in large model spaces have shown that the theoretical $M 1$ strengths reproduce the experimental values; the quadrupole transitions, however, are still seriously underestimated, even in the largest model spaces.

The two-body cluster approximation is the main difference between this approach and a previous NCSM calculation that reported effective charges for ${ }^{6} \mathrm{Li}$ in agreement with the phenomenological charges usually employed in shell model calculations. Thus, in Ref. [2], the authors have performed a Lee-Suzuki transformation which includes up to six-body correlations, by transforming the Hamiltonian from an initial $6 \hbar \Omega$ space to a $0 \hbar \Omega$ model space, equivalent to a core calculation which fixes four particles in the $0 s$ shell. The electromagnetic operators which they obtained were reproducing the transition strengths obtained with bare operators in $6 \hbar \Omega$, considered the correct values. This suggest that the higher order clusters are important for renormalization of electromagnetic operators. 


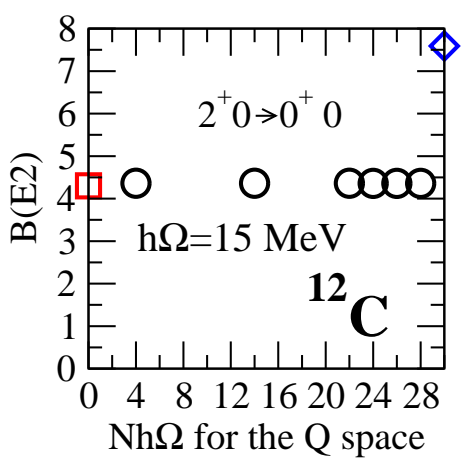

Fig. 1. $B(E 2)$ in ${ }^{12} \mathrm{C}$ using effective interaction derived from AV8' potential [7]. Results as a function of the dimension of the $Q$-space included (circles) are compared with the bare operator (square) and the experimental value (diamond).

By using a Gaussian operator of variable range, we can show that the renormalization, obtained at the two-body cluster level [4], depends strongly upon the range of the operator [8]. Thus, for short range operators, such as the relative kinetic energy, the renormalization is strong, while for long range operators, it is very weak. The quadrupole operator is infinite range and therefore very weakly renormalized at the two-body cluster level.

In summary, we have implemented the Lee-Suzuki procedure for the renormalization of general one- and twobody operators, at the two-body cluster level. The renormalization is much more involved than for the Hamiltonian, so that we include in the renormalization states from the excluded space one shell at a time, observing the convergence of matrix elements. We have shown that the renormalization fails to improve on the transition strengths obtained with bare operators, and we suggest that this is due to the two-body cluster approximation which renormalizes short range correlations; the electromagnetic multipoles, however, are infinite range and thus very weakly renormalized.

I.S. and B.R.B acknowledge partial support by NFS grants PHY0070858 and PHY0244389. The work was performed in part under the auspices of the U. S. Department of Energy by the University of California, Lawrence Livermore National Laboratory under contract No. W7405-Eng-48. P.N. received support from LDRD contract 04-ERD-058. J.P.V. acknowledges partial support by USDOE grant No DE-FG-02-87ER-40371.

\section{References}

1. P. J. Ellis and E. Osnes, Rev. Mod. Phys. 49, 777 (1977).

2. P. Navrátil, M. Thoresen, and B. R. Barrett, Phys. Rev. C 55, R573 (1997).

3. K. Suzuki and S.Y. Lee, Prog. Theor. Phys. 64, 2091 (1980); K. Suzuki, Prog. Theor. Phys. 68, 246 (1982).

4. P. Navrátil, J. P. Vary, and B. R. Barrett, Phys. Rev. C 62, 054311 (2000).
5. P. Navrátil, H. Geyer, and T. T. S. Kuo, Phys. Lett. B 315, 1 (1993).

6. I. Stetcu, B. R. Barrett, P. Navrátil, and C. W. Johnson, submitted to Int. J. Mod. Phys. G. [arXiv:nucl-th/0409072]

7. R. B. Wiringa, V. G. J. Stoks and R. Schiavilla, Phys. Rev. C 51, 38 (1995)

8. I. Stetcu, B. R. Barrett, P. Navrátil, and J. P. Vary, to be submitted. 